\title{
Improving Logistics and Waste Management for Deep Space Human Exploration
}

\author{
Michael K. Ewert, ${ }^{1}$ and James Lee Broyan, Jr. ${ }^{2}$ \\ NASA Johnson Space Center, Houston, Texas 77058
}

\begin{abstract}
NASA's Advanced Exploration Systems Logistics Reduction Project is developing technologies that reduce mission mass and volume for exploration. Recently there has been increasing interest in determining the quantity of consumable logistics and system spares necessary to ensure a certain level of reliability. This is influenced by a technology's criticality and degree of impact to the overall mission. Technologies that directly reduce mass (e.g. longer wear crew clothing) are relatively straightforward for calculating the savings and understanding the mission impacts. Waste management technologies that process waste can reduce mass, but spares and contingency modes are more interwoven with other vehicle systems, so assessment is more complex. This paper considers mission benefits while also considering impacts from hardware failures for technologies including: crew clothing, reusable cargo bags for habitat outfitting, automated RFID cargo tracking, trash processing/storage/repurposing, and high reliability toilets.
\end{abstract}

\section{Nomenclature}

ACS $=$ Advanced Clothing Systems

AES $=$ Advanced Exploration Systems

$A L M=$ Autonomous Logistics Management

$C T B=$ Cargo Transfer Bag

ESM = Equivalent System Mass

$H M C=$ Heat Melt Compactor

$I S S=$ International Space Station

$L E O \quad=$ Low Earth Orbit

$L R=$ Logistics Reduction (project)

$M C T B=$ Multi-purpose Cargo Transfer Bag

REALM = RFID Enabled ALM

$R F \quad=$ Radio frequency

$R F I D=$ Radio Frequency Identification

SBIR = Small Business Innovative Research

SysML = System Modeling Language

$T t G \quad=$ Trash-to-gas

$T t S G \quad=$ Trash-to-supply-gas

$U H M W P E=$ Ultra-high-molecular-weight polyethylene

$U W M S=$ Universal Waste Management System

\section{Introduction}

TASA is advancing technologies needed to explore the moon and Mars through its Advanced Exploration Systems 1 (AES) program. For long human missions such as these, high reliability and availability of crew support systems is key. The AES Logistics Reduction (LR) project is working to optimize portions of the logistics supply chain and

\footnotetext{
${ }^{1}$ Life Support Systems Analyst, Crew \& Thermal Systems Division, EC2, JSC, 2101 NASA Pkwy., Houston, TX, not AIAA affiliated.

${ }^{2}$ AES Logistics Reduction Project Manager, Crew \& Thermal Systems Division, 2101 NASA Parkway, Houston, TX, 77058/Mail Stop EC7, AIAA member.
} 
waste disposal strategies since the efficiency and robustness of these mission elements is paramount. The total resource impact of a given technology encompasses its mass, volume, power and crew time requirements as well as the spare parts and consumable items needed to keep it running for the duration of a mission. Owens has shown that the spare parts required to achieve acceptable reliability levels can be significant for many spacecraft systems, so the LR project has begun to address this issue during technology development $[1,2,3]$.

Previous analysis has shown the net mission benefits of several of the LR technologies without consideration of redundancy or spares [4]. This paper provides a brief project update on the LR technologies, new analysis for two of the LR technologies to account for required spares, and general updates to the mission benefits for deep space exploration. The Global Exploration Roadmap [5] outlines a Deep Space Gateway built up over a series of flights in cislunar space. Initially, it will be visited by a crew of four for 30 days at a time. These missions may increase in frequency and length as the Gateway evolves and additional transportation systems become available. The Gateway will provide communication between the Earth and moon, serve as a staging point for lunar and Mars missions and provide valuable operational data on habitation technologies intended for long duration space exploration. This includes logistics delivery and tracking systems and waste reuse and disposal technologies. Beyond the Gateway, a human transit to Mars will require logistics and waste strategies and technologies to be efficient, robust and crewfriendly for this long endurance mission. While many of the LR technologies will be beneficial even on shorter stays at the Gateway, their larger impact will be for longer exploration missions. Thus, the benefits analysis below focuses on a 1000-day class mission, such as a human mission to Mars and back.

\section{Logistics Reduction Project Technologies}

\section{A. Universal Waste Management System}

The Universal Waste Management System (UWMS) is a new compact commode (Fig. 1) for use on ISS, Orion, and future human spacecraft [6]. The International Space Station (ISS) UWMS version uses a precision urine pretreatment dose pump and conductivity sensor to ensure the urine is stabilized prior to treatment by the ISS Urine Processor Assembly. Orion will utilize chemical tablets to prevent precipitates from forming before it vents the urine overboard. Future exploration vehicles being developed by NASA have smaller habitable volumes than the ISS, and as habitable volumes decrease, so must the toilet hardware. UWMS was designed to be more compact through the use of a dual-fan-rotary-separator and concentric odor-bacteria filter. The core UWMS is being developed by United Technologies Corporation Aerospace Systems (UTAS). Integration hardware for ISS is being developed by NASA and Boeing Aerospace. The UWMS is currently scheduled to be installed on the ISS in the fall of 2019 and fly on the Orion EM-2 flight.

Advanced Fuel Research, Inc, is researching torrefaction of feces under an SBIR Phase II award. The goal is to process collected feces and reduce the number of UWMS fecal canisters required for an exploration mission [7]. The initial spares for ISS have been identified and a spares mass assessment for exploration will be published in the future.

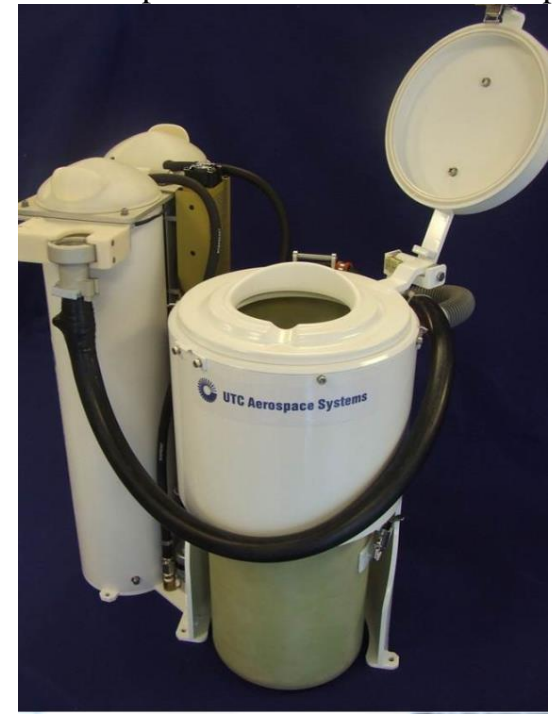

Fig. 1 UWMS prototype with fecal collection system and urine tanks (tanks removed from final design) 


\section{B. Heat Melt Compactor}

The Heat Melt Compactor (HMC) is a device for reducing trash volume and stabilizing trash for long-term storage. HMC will also recover water from waste materials and produce microbiologically stable, low-volume, tiles that can be used to augment radiation protection, or else easily stored or disposed. For a one-year mission of four-crew, it is estimated that HMC could recover 7-10 cubic meters of habitable volume, produce over $900 \mathrm{~kg}$ of radiation shielding tiles, and recover $250 \mathrm{~kg}$ of water from $\sim 1,300 \mathrm{~kg}$ of trash. A full-scale, second-generation (Gen2) ground prototype HMC has been developed by NASA's Ames Research Center. This unit is being used for ground tests to develop process parameters, discover challenges with the technology and reduce risk for flight unit development. A recent focus has been to study the possibility of a vent-to-vacuum option, with and without water-save [8]. The intent of the vent-to-vacuum approach is to reduce the amount and complexity of contaminants in the gas and water recovery systems to reduce overall complexity, mass, and volume and to inform future exploration technology procurements. The Gen 2 HMC system component layout is used for initial spares calculations discussed later in the paper.

In July 2018 NASA announced a Next Space Technologies for Exploration Partnerships (NextSTEP) Broad Agency Announcement, Appendix F: Logistics Reduction in Space by Trash Compaction and Processing System (TCPS) [9]. TCPS includes HMC and related volume reduction technologies in a two step competitive procurement activity based on system performance. In the first step, basic trash compaction and stabilization technologies are required and gas or water recovery is optional (See Fig. 2). The intent is to identify robust technology approaches that provide the most benefit and are likely to be reliable as demonstrated by preliminary design prototypes. The second step of procurement will select the most successful approaches and develop them to ISS flight demonstration fidelity for a flight test in $\sim 2022$. Predictions of required spares and mission benefits will be updated as the TCPS technology develops over the next three years.

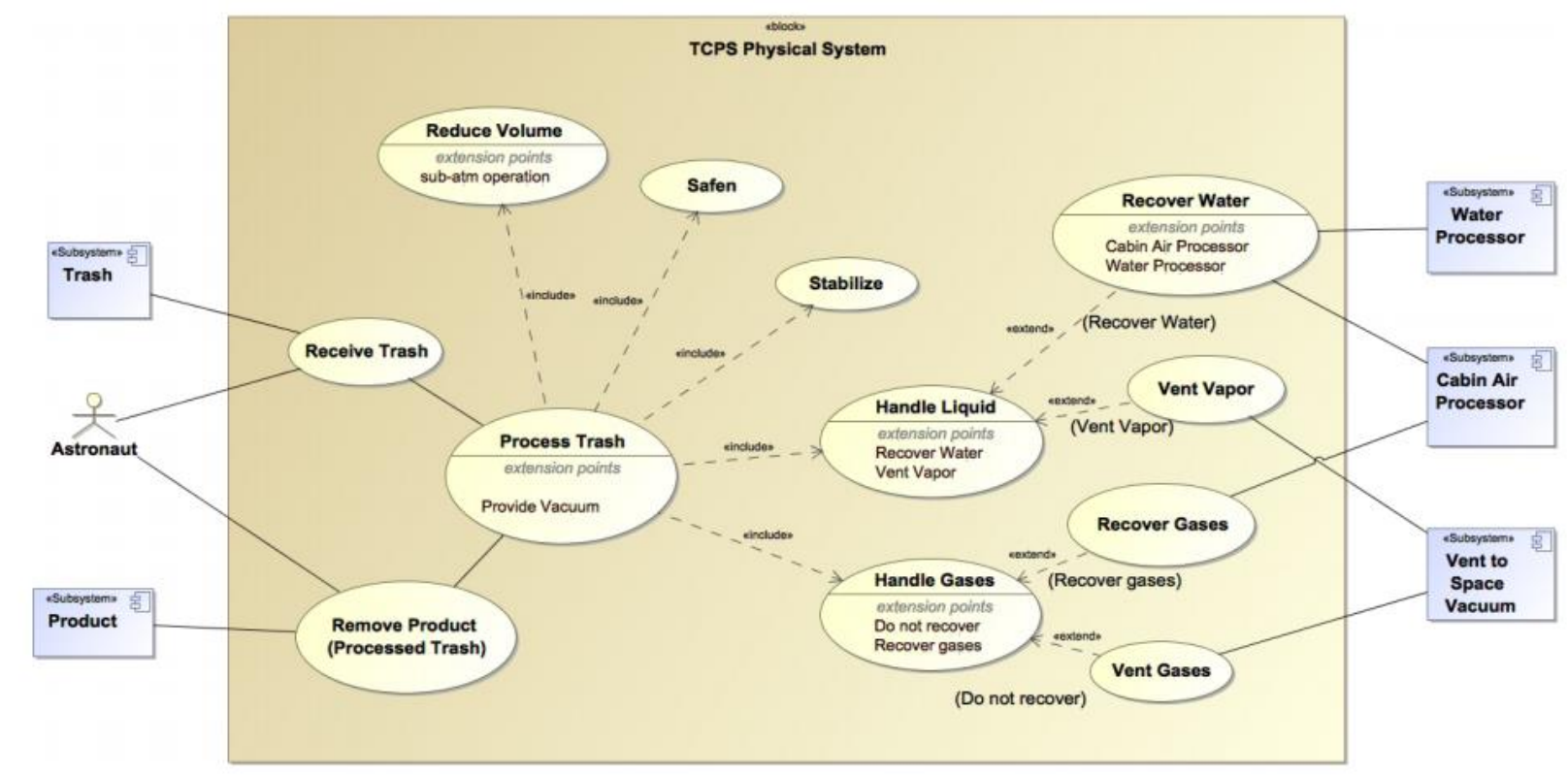

Fig. 2 SysML illustration of notional TCPS system showing requested and optional functions.

\section{Trash-to-Gas}

The idea of reducing trash and other waste products to their elemental composition for disposal and/or reuse is often called Trash-to-Gas (TtG), or Trash-to-Supply-Gas (TtSG) in the case of gas reuse. From 2012-2014, the LR project investigated five different thermochemical processes for $\mathrm{TtG}$ and $\mathrm{TtSG}$ and performed a trade study comparing the options [10]. Aside from the various thermal process options such as incineration shown in Fig. 3, the technology could be developed to optimize resource recovery, such as methane for propulsion, or optimized for waste disposal with the resulting gases simply vented from the spacecraft. An intermediate case could use product gases in resistojet thrusters. When waste products are disposed overboard, there can sometimes be a significant propulsion advantage due to reduced spacecraft mass [11]. 

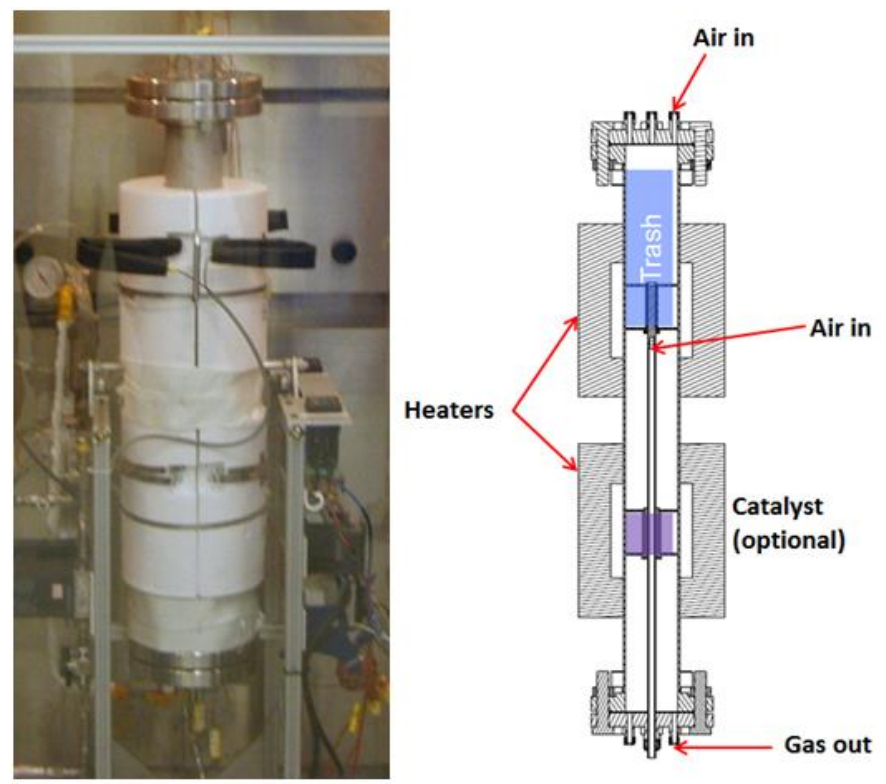

Fig. 3 Incineration/Gasification System at Kennedy Space Center

Although TtG research under the LR project largely ended in 2015, LR has continued to be an advocate for its development under various NASA innovation initiatives. Over the past year, the Space Technology Mission Directorate has funded the Orbital Syngas/Commodity Augmentation Reactor (OSCAR) at Kennedy Space Center. In cooperation with OSCAR, AES LR is conducting a crowd source challenge later this year to design a feed mechanism to move trash into the hot zone of a TtG reactor. LR is also in discussions with the OSCAR team on future technology demonstrations because $\mathrm{TtG}$ has the potential to reduce overall transit vehicle logistics mass.

\section{Advanced Clothing Systems}

The goal of Advanced Clothing Systems (ACS) tasks is to directly reduce the mass and volume of clothing to be launched. ACS will directly reduce up-mass and disposal burdens. These benefits will enhance long-duration missions beyond low-Earth orbit (LEO). Clothing accounts for a significant portion $(\sim 6 \%)$ of the logistical mass on current human missions. Since no space laundry is available, the clothing becomes trash when it is too dirty. Advanced lightweight and antimicrobial fabrics that are currently used in some commercial off-the-shelf (COTS) garments were previously evaluated, both on ground and on ISS, for extended use during long-duration missions [12, 13]. By carefully selecting the best COTS fabrics, use of light-weight and longer-wear clothing can increase the break-even point for laundering vs. disposable clothing, allowing the delay of full laundry development until missions exceed approximately 12 months. Since Mars transit missions are currently envisioned to be 6-9 months in duration, this means full water based laundry with its challenging phase separation issues does not need to be developed. As an intermediary step toward laundry, NASA has performed initial evaluations of clothes freshening technologies in the area of no- or low-water cleaning to extend clothing use for transit missions. Initial results have been mixed. While unacceptable odors may be addressed, dander and surface soiling have not been satisfactory addressed to date.

\section{E. Multi-purpose Cargo Transfer Bags}

Multi-purpose Cargo Transfer Bags (MCTBs) have been designed to repurpose the cargo transfer bags (CTBs) used on ISS for on-orbit outfitting. The MCTB task converts cargo bags into useful crew items on-orbit after they have provided their initial logistics function. MCTBs can be used for constructing crew quarters, privacy or soundabsorbing partitions, contingency water storage, wastewater processing units, or other purposes. Reuse of MCTB logistics carriers reduces the need to fly additional items and decreases the volume of trash. By repurposing MCTBs, some dedicated crew items do not have to be launched and the overall mission mass is decreased. For non-LEO missions, the vehicle interior volume will be relatively fixed. MCTBs will enable this volume to be used more effectively through reuse and rearrangement of logistical components.

In September 2016, four acoustic MCTBs were flown and deployed around the ISS node 3 treadmill. The installed MCTBs are shown in Fig. 4. As expected, the Acoustic MCTBs provide 2 dBA ( 37\% reduction) of sound mitigation 
during treadmill use. The MCTBs are installed next to the ISS Waste Handling Compartment (WHC) cabin and, combined with the treadmill exercise, this is a high moisture and potential soiling environment. The MCTBs are still in use on-orbit and crew evaluation and microbial sampling of them continues. The results of this experiment will help determine how frequently MCTBs would need to be replaced (spared) for exploration missions.

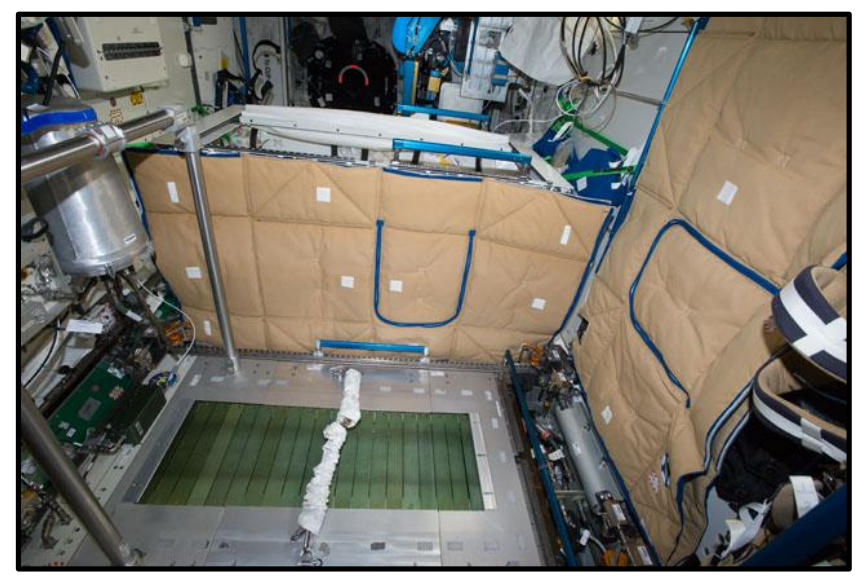

Fig. 4 Acoustic MCTBs (tan color) installed in Node 3 next to treadmill (green running surface on bottom).

\section{F. Radio Frequency Identification Enabled Autonomous Logistics Management}

In addition to the well-known subset of functions pertaining to automated localization and inventory management of all physical assets within a vehicle, Autonomous Logistics Management (ALM) is a broad area that also includes propellant delivery and vehicle-level architectures. Radio Frequency Identification (RFID) Enabled Autonomous Logistics Management (REALM) focuses on a subset of ALM functions utilizing RFID technologies [14]. RFID tags attached to logistical items can be read electronically with antennas strategically located around the spacecraft or on mobile platforms (a.k.a. free-flyers). This capability has the potential to dramatically reduce crew time spent on general inventory management and searching for lost items, and to increase packing volume efficiencies by assuring that the crew can find even densely packed items.

The problem of locating all mission items within and around a vehicle are complicated by many factors, including the preference to rely only on passive (unpowered) tags, restrictions on RF transmit power, layered storage of logistics, the challenging RF scattering environment of vehicles, and metallic storage enclosures. To address this complex problem, associated RFID technologies are categorized into three classes:

- Dense Zone technologies - enclosures with conductive, or shielded, boundaries and an integrated RFID reader to interrogate the items contained within.

- Sparse Zone technologies - open areas of a habitat module outside of dense zones.

- Complex Event Processing (CEP) - "smart" applications, to infer item locations based on context from the sparse and dense zone technologies.

In February 2017, REALM-1 deployed 6 RFID readers and 24 antennas on ISS. Data from over 8 billion tag reads has been successfully collected and relayed to the ground. Three NASA and University of Massachusetts localization algorithms have been developed, and a fourth is in work. Although still in the 2-year experimental phase, REALM-1 has already been used to assist ISS in the search for $~ 100$ missing items. REALM was able to provide some level of assistance for $60-70 \%$ of the requests. Attempts for which no tag reads were found are believed to correlate largely with cargo outside of REALM-1 instrumentation, cargo buried or otherwise obscured before REALM-1 was activated, or cargo that was unloaded while REALM-1 was unavailable. There has been very high read accuracy as cargo is offloaded from visiting vehicles. A screen shot tracking a CTB from left to right is shown in Fig. 5.

A mobile version of the technology is under development as REALM-2 and will be a payload on the next generation freeflyer 'Astrobee'. A one-year REALM-2 ISS experiment is planned for Fall 2019. The dense zone configuration, REALM-3, is in the initial design phases and will fly to ISS in 2020. Based on the performance of the REALM systems the logistics tracking configuration for exploration will be determined and reported in the future. 


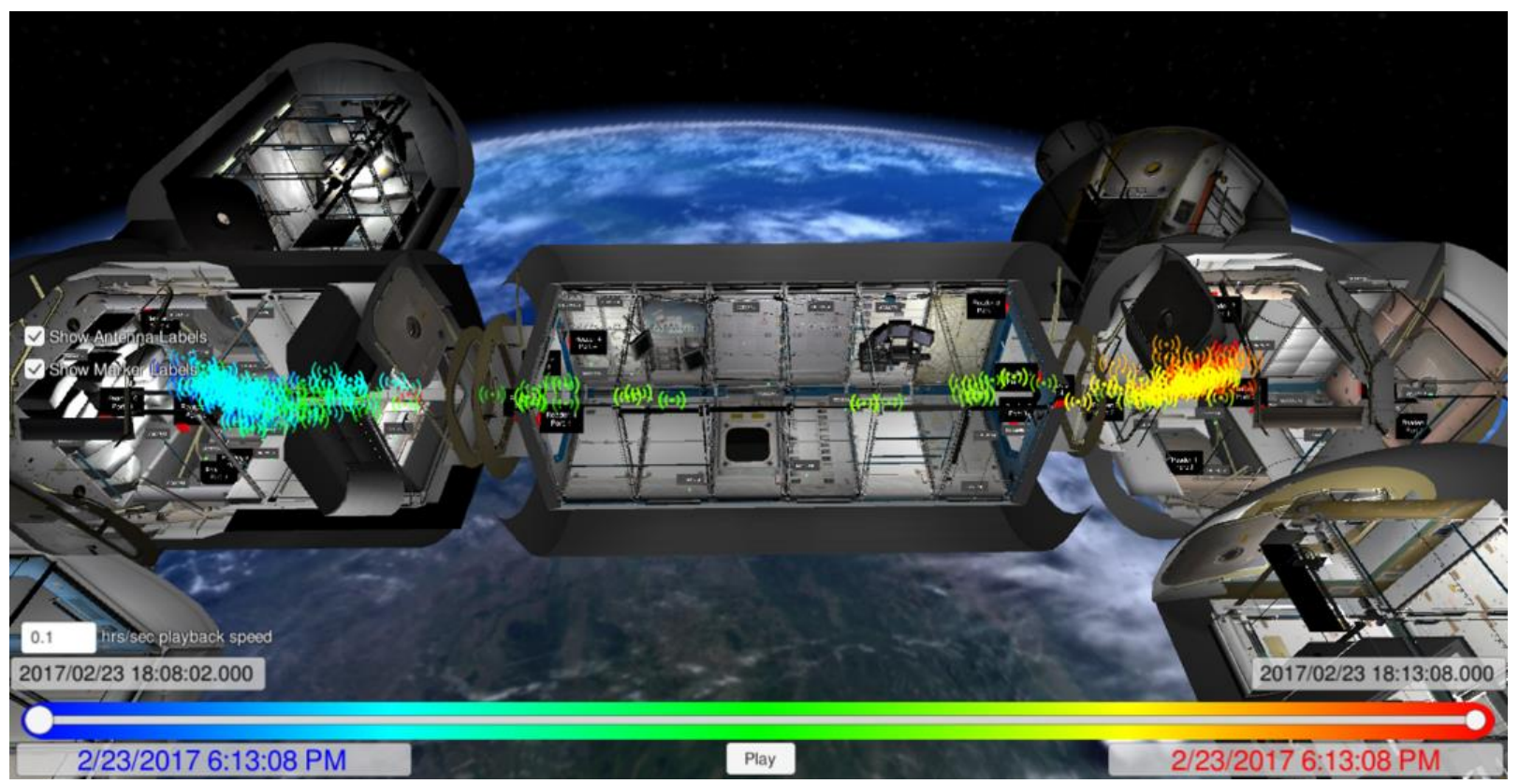

Fig. 5 REALM tracking of a cargo bag during SpX10 unloading and moving through node 2, US Lab, and node 1 over a 5 minute period.

\section{G. Autonomous Logistics Management}

Autonomous Logistics Management (ALM) also includes addressing how to effectively manipulate and translate logistics prior to crew arrival and after crew departure on an Earth-independent habitat. The concept of operations for many future exploration missions involves spacecraft that are periodically crewed and otherwise dormant. Depending on the mission, it is likely that cargo vehicles will arrive and depart during the dormant periods. ALM combines sensing systems like REALM and Astrobee with mobile manipulations systems (e.g., Robonaut, Mantis) to assess and configure the vehicle's logistics when crew is not available. This autonomous capability could also be utilized to support mission phases when the crew is present, but this has not been the focus of AES LR.

The Logistics project has performed a feasibility assessment of robotic manipulation of logistics. An end-to-end CTB retrieval task was attempted, which involved robotic translation, manipulating and opening a hatch, positioning in front of the CTB, and removing the CTB from its stowage position (Fig. 6). It is important to note that Robonaut is not being driven by an operator; it is self-planning the task and motion. The Robonaut 2 IVA mobility system was used as a test platform for developing the software algorithms needed to achieve this task, but is a generic planning tool applicable to other generic agents. The most notable development was the integration of constraints into the motion planning problem. These constraints could involve the objects that had to be manipulated (e.g. the hatch can move in a single planar direction), or involve the state space constraints of the robot (e.g. robotic attach points on the handrails and axis constraints due to the gravity offload facility). The result is a general algorithm, replacing previous custom versions, which can plan online while accounting for multiple constraints.

Current development efforts include the collaboration between multiple robotic agents to locate a missing logistics item with REALM-2 as a payload directing Astrobee using a homing function. Then REALM-2 would use Astrobee's position information to inform Robonaut to translate to the location and retrieve the CTB. Continued development will lead to an ISS demonstration of Robnaut, REALM, and Astrobee in the 2020 timeframe. 


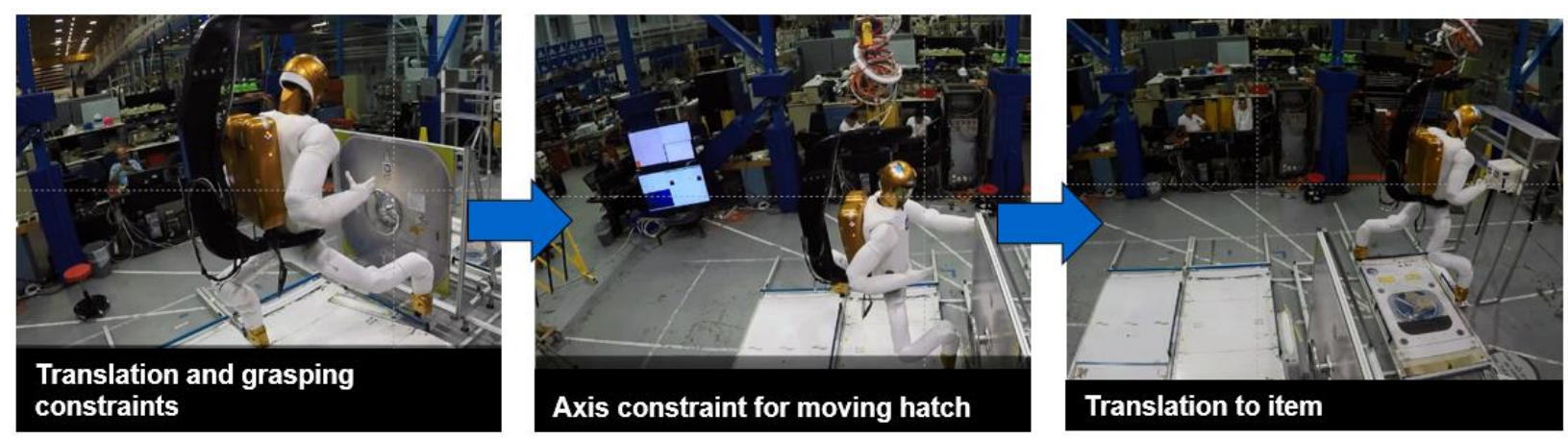

Fig. 6. Robotic operation of hatch and retrieval of cargo bag using autonomous constrained motion planning.

\section{Mission Benefits Analysis}

As described in reference [4], mass, power, volume and crew time savings can be quantified for the technologies described above. To be fair, these benefits must be balanced against the additional resource requirements [mass, power, volume, crew time] that the technology may require. By using an equivalent system mass (ESM) technique, the net savings can be calculated and normalized to launch mass savings [15]. Below, these net mission benefits are calculated, and for the first time required spare parts were estimated and included for two of the waste processing technologies. The ESM conversion factors used in the analysis were derived from reference [16] and are listed in Table 1.

When it comes to waste disposal systems, mission benefits of various technology options may extend well beyond hardware mass or volume savings. Human factors are critical for systems that have the potential to expose the crew to discomfort or even hazards. Also, as demonstrated in reference [11], removal of waste from the spacecraft during a mission can have both positive effects such as propellant savings and negative effects such as reduced radiation protection. These factors are not directly accounted for in the technology benefits analysis below, but are being seriously considered by the development teams.

Mission benefits for each of the LR technologies are described and quantified below in the context of a 1000-day class human exploration mission, such as a round trip to Mars with a crew of four. While such a mission would likely include a surface stay using one or more additional spacecraft, the analysis is simplified as 1000 days in the Mars transit vehicle. This could actually be a realistic contingency scenario in case the Mars lander or surface habitat failed.

Table 1 Equivalent system mass resource conversion to launch mass for Mars transit vehicle.

\begin{tabular}{|c|c|}
\hline ESM volume factor $(\mathrm{kg} / \mathrm{m} 3)$ & 29.5 \\
\hline ESM power factor $(\mathrm{kg} / \mathrm{kW})$ & 41 \\
\hline ESM thermal factor $(\mathrm{kg} / \mathrm{kW})$ & 55.4 \\
\hline ESM crew time factor $(\mathrm{kg} / \mathrm{hr})$ & 0.8 \\
\hline
\end{tabular}

\section{A. Universal Waste Management System}

UWMS will be more compact through the use of a dual-fan-rotary-separator and concentric odor-bacteria filter. The UWMS design is being finalized, and while the initial spares have been identified, the ESM benefits have not yet been completed at the time of this paper. The benefits analysis will compare the UWMS spares and consumables to the current ISS Waste and Hygiene Compartment's toilet system. One major factor in UWMS consumables estimates will be the way compaction occurs because it directly impacts the defecations per canister and hence the total number of canisters needed for a mission. Compaction tests are planned with flight like canisters that have recently been fabricated. This data will be used in providing mission benefit analysis in the future. The benefits of possible fecal processing to recover water as well as reduce UWMS fecal canisters will also be analyzed. These results should be published in 2019. Flight fecal canisters will be returned from ISS in early 2020 to determine in-flight compaction efficiency to improve estimates for exploration. 


\section{B. Heat Melt Compactor}

Primary benefits of the HMC are compacting trash to reduce storage volume and stabilizing the resulting product for long-term storage. Depending on the design, water can also be recovered for reuse. Yet another benefit, that may or may not result in mission mass savings, is reuse of the product as radiation shielding. As discussed in reference [17], minimum radiation standards may be met using other on-board spacecraft items. Thus, cases including and excluding this benefit are included below. Nevertheless, if reusable tiles are produced by the HMC, they will always contribute to the "as low as reasonably achievable" (ALARA) principle for crew radiation exposure.

Table 2 shows the resource "costs" of the HMC based on previous NASA technology development [8]. These include the mass and volume of the device and incremental mass of the power and thermal control system capacity required to support HMC operation. Additionally, the crew time required to operate the system is accounted for by estimating the additional resources used by the crew per work hour in space. The ESM factors that translate volume and power into equivalent mass are also shown in Table 2, along with some of the ESM calculations.

Table 2 Costs and benefits of Heat Melt Compactor.

\begin{tabular}{|c|c|c|c|c|}
\hline HMC mass $(\mathrm{kg})$ & 120 & & & \\
\hline HMC volume $\left(\mathrm{m}^{3}\right)$ & 0.2 & & ESM volume factor $(\mathrm{kg} / \mathrm{m} 3)$ & 29.5 \\
\hline HMC peak power $(\mathrm{kW})$ & 0.5 & & ESM power factor $(\mathrm{kg} / \mathrm{kW})$ & 41 \\
\hline HMC peak thermal $(\mathrm{kW})$ & 0.5 & & ESM thermal factor $(\mathrm{kg} / \mathrm{kW})$ & 55.4 \\
\hline Crew operation time (hr/day) & 0.2 & & ESM crew time factor $(\mathrm{kg} / \mathrm{hr})$ & 0.8 \\
\hline "Costs" & & & & \\
\hline HMC initial ESM (-kg) & \multicolumn{3}{|c|}{$120+0.2 * 29.5+0.5 * 41+0.5 * 55.4=174$} & \\
\hline Crew operation ESM (-kg/day) & \multicolumn{2}{|c|}{$0.2 * 0.8=0.16$} & & \\
\hline Savings & & & Notes & \\
\hline Volume recovery rate $\left(\mathrm{m}^{3} / \mathrm{CM}-\mathrm{d}\right)$ & 0.0063 & & $7: 1$ & \\
\hline Water recovery rate $(\mathrm{kg} / \mathrm{CM}-\mathrm{d})$ & 0.1704 & & From trash only (no brine) & \\
\hline Radiation shield prod. rate (kg/CM-d) & 0.6621 & & $90 \%$ as effective as UHMWPE & \\
\hline Volume savings ESM (kg/day) & \multicolumn{2}{|c|}{$0.0063 * 4 * 29.5=0.743$} & For 4 crewmembers & \\
\hline Water savings ESM (kg/day) & \multicolumn{2}{|c|}{$0.1704 * 4=0.682$} & For 4 crewmembers & \\
\hline Radiation shield savings ESM (kg/day) & \multicolumn{2}{|c|}{$0.6621 * 4=2.648$} & For 4 crewmembers & \\
\hline
\end{tabular}

\section{Consideration of Spares:}

Previous LR benefits analysis was done without considering hardware redundancy or reliability. In order to get a more realistic picture of the resource "costs" of HMC, a spare parts analysis was added as an additional case. Fig. 7 shows the net resource savings due to HMC versus days of mission duration for 4 cases: with and without a mass credit for the radiation shielding tiles produced and with and without adding spares to the costs. The spares analysis was performed using NASA's Exploration Maintainability Analysis Tool (EMAT), which is described in reference [1]. The HMC spare parts added $175 \mathrm{~kg}$ for the 1000 day duration at a mission reliability of $99 \%$. This shifted the point at which benefits outweigh costs from 138 to 225 mission days when radiation shielding benefits are not included. The HMC technology breaks even much sooner if shielding benefits are included. Not all mission scenarios require additional passive shielding, so mission specific benefits are likely between the two curves. 


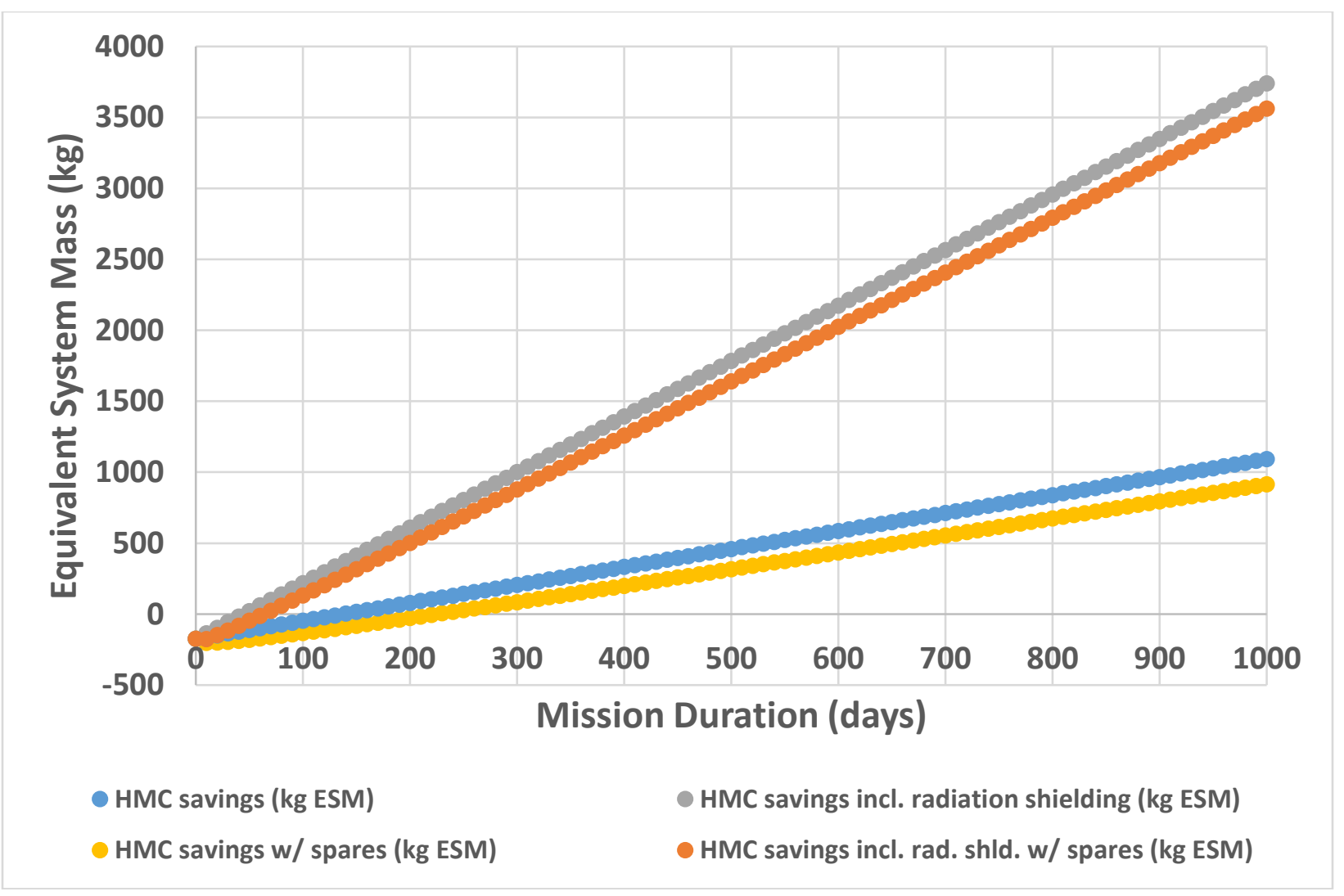

Fig. 7 Heat Melt Compactor net ESM benefits versus mission duration for several cases.

\section{Trash-to Gas}

The TtG venting case is considered in this benefits analysis; however, depending on mission architecture and objectives, producing gases for resistojets or methane for fuel may result in greater benefits. The resource "costs" and benefits of TtG hardware and operation were summed in a manner similar to the HMC analysis. Table 3 contains data used in the ESM calculations. Estimates were based on a steam reformation process capable of processing $6.25 \mathrm{~kg} / \mathrm{day}$ of trash, feces and urine brine.

Table 3 Costs and benefits of Trash-to-gas venting case.

\begin{tabular}{|l|l|l|l|l|}
\hline TtG mass $(\mathrm{kg})$ & 150 & & & \\
\hline TtG volume $\left(\mathrm{m}^{3}\right)$ & 0.2 & & ESM volume factor $(\mathrm{kg} / \mathrm{m} 3)$ & 29.5 \\
\hline TtG peak power $(\mathrm{kW})$ & 1.78 & & ESM power factor $(\mathrm{kg} / \mathrm{kW})$ & 41 \\
\hline TtG peak thermal $(\mathrm{kW})$ & 0.74 & & ESM thermal factor $(\mathrm{kg} / \mathrm{kW})$ & 55.4 \\
\hline Crew operation time $(\mathrm{hr} /$ day) & 0.1 & & ESM crew time factor $(\mathrm{kg} / \mathrm{hr})$ & 0.8 \\
\hline Savings & & & Notes & \\
\hline Volume recovery rate $\left(\mathrm{m}^{3} / \mathrm{CM}-\mathrm{d}\right)$ & 0.0079 & & & \\
\hline Water recovery rate $(\mathrm{kg} / \mathrm{CM}-\mathrm{d})$ & 0.197 & & From trash, feces, brine & \\
\hline Volume savings ESM $(\mathrm{kg} /$ day) & $0.0079 * 4 * 29.5=0.94$ & For 4 crewmembers & \\
\hline Water savings ESM $(\mathrm{kg} /$ day) & $0.197 * 4=0.786$ & For 4 crewmembers & \\
\hline
\end{tabular}


Consideration of Spares:

Again, similar to the HMC analysis, a spare parts assessment was made to achieve mission reliability of 99\%. Fig. 8 shows the net ESM launch mass savings versus mission duration, with and without considering spares. As expected, the net benefit is less when spares are considered, assuming that the competing approach (e.g. trash storage) did not need any contingency assets. It is important to note that these savings do not include the fuel saving benefits that may also accrue when spacecraft mass is reduced before a propulsive maneuver. Those savings will depend on the vehicle and mission architecture.

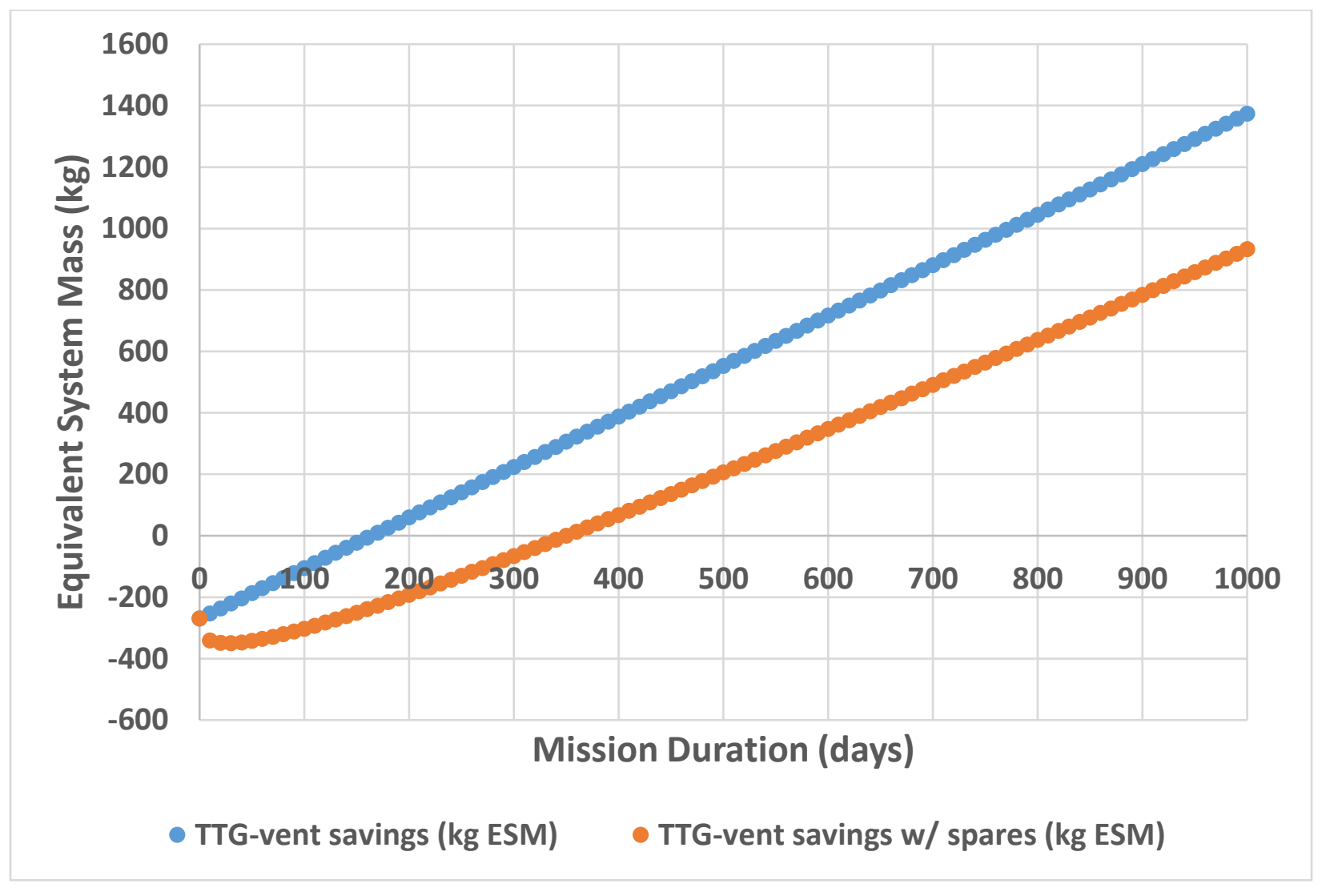

Fig. 8 Trash-to-gas venting case net ESM benefits versus mission duration.

\section{Advanced Clothing Systems}

As mentioned above, the first benefits of ACS are direct mass and volume reduction of disposable clothing due to lighter weight, longer wear fabrics. Future updates will include results from investigation of low-water-use freshening techniques as well as the benefits of a water-based laundry for longer missions. For now, the ACS benefits are estimated based on substitution of lower mass fabrics for those currently used in ISS clothing, which is worn as long as possible before being thrown away. ACS studies have shown wool and mod-acrylic to be good candidates to replace some cotton and polyester garments currently worn on ISS $[12,13]$. Table 4 shows estimated potential savings with ACS clothing versus current ISS clothing for two mission durations.

Table 4 Potential savings due to an advanced clothing system.

\begin{tabular}{|l|r|r|}
\hline Mass $(\mathrm{kg})$ & 365 days & 1000 days \\
\hline Volume $\left(\mathrm{m}^{3}\right)$ & 82 & 224 \\
\hline
\end{tabular}




\section{E. Multi-purpose Cargo Transfer Bags}

The benefit of using MCTBs is direct launch mass savings for any required items that can be eliminated in favor of reusing a cargo bag for the intended purpose. Leading ideas for reuse of MCTBs are as room partitions, sound absorbing blankets and private crew quarters. Table 5 shows a rough estimate of potential mass and volume savings for these three use cases based on mass and volume of bags reused. These estimates were recently updated to third design generation CTB mass values based on ISS state-of-the-art bags. MCTB designs have not been updated since 2015, and additions to the bags that may be required for reuse were not included. A more accurate indication of savings (future work) would be to estimate the mass and volume of hardware that would have been designed specifically for each purpose.

Table 5 MCTB potential mass and volume savings in an exploration habitat with $\mathbf{4}$ crew members.

\begin{tabular}{|l|c|c|c|}
\hline Repurposing potential & \# of MCTB's & $\begin{array}{l}\text { Potential mass } \\
\text { savings }(\mathrm{kg})\end{array}$ & Potential volume savings (m $\left.{ }^{3}\right)$ \\
\hline Room partitions & 9 & 8.1 & 0.06 \\
\hline Sound absorbing blankets & 4 & 3.6 & 0.03 \\
\hline Crew quarters & $16^{*} 4=64$ & 57.6 & 0.41 \\
\hline TOTAL & $\mathbf{7 7}$ & $\mathbf{6 9 . 3}$ & $\mathbf{0 . 5}$ \\
\hline
\end{tabular}

\section{F. Radio Frequency Identification Enabled Autonomous Logistics Management}

A key benefit of the REALM technology is reduction of crew time spent looking for items they need to live or work. Based on ISS experience, about 34 hours per year are spent looking for items. It was assumed that this could be reduced by $80 \%$ for items located by REALM and that tag read accuracy is $90 \%$. An additional time savings of 13 hours per year was estimated for periodic inventories. The resultant crew time savings are calculated on an annual basis in Eq. 1. On ISS, REALM has also helped to correct the inventory database, thus preventing additional items from becoming lost.

$$
\text { Estimated savings per year }=34 \mathrm{hr} / \mathrm{yr} * 0.80 * 0.90+13 \mathrm{hr} / \mathrm{yr}=37 \mathrm{hrs} / \mathrm{yr}
$$

Another benefit of REALM is that CTBs can now be packed for volumetric efficiency rather than for crew efficiency in locating items. It was estimated that void space in CTBs can be decreased from $25 \%$ to $5 \%$, thus eliminating $20 \%$ of required CTB mass and volume. Again, the third generation CTB mass was used to arrive at estimated savings of $14 \mathrm{~kg}$ and $0.83 \mathrm{~m}^{3}$ for bags containing crew consumables, EVA and health supplies. Mass, volume and power for a complete REALM system on the transit vehicle were estimated to be $46.1 \mathrm{~kg}, 0.002 \mathrm{~m}^{3}$ and $39 \mathrm{~W}$. Summing the REALM savings and resource 'costs' in ESM for a one year period results in $68 \mathrm{~kg}$ and $-50 \mathrm{~kg}$, respectively. The net benefit will increase with mission duration.

\section{G. Autonomous Logistics Management}

An ESM analysis of autonomous logistics management has not yet been conducted. The primary benefits of ALM will be crew time savings and the ability to accomplish tasks while the crew is not present in both planned and contingency scenarios. Benefits analysis will depend heavily on the particular mission, vehicle and robotic assets.

\section{Conclusion}

The AES LR project is developing many new technologies to support NASA's deep space human exploration missions. These technologies are designed to reduce logistics resupply from Earth and transform waste products from a problem to a resource opportunity. A benefits analysis was performed to quantify many of the resource savings and costs associated with these technologies. Using ESM analysis, the net savings in launch mass was calculated versus mission duration for a transit habitat with 4 crew members. Fig. 9 is a summary graph of the savings, highlighting specific options for some of the technologies (e.g. TtG-venting). Spares are not included in Fig. 9 for consistency between technologies. This paper also discussed additional mission benefits that could not be captured in a single graph. 


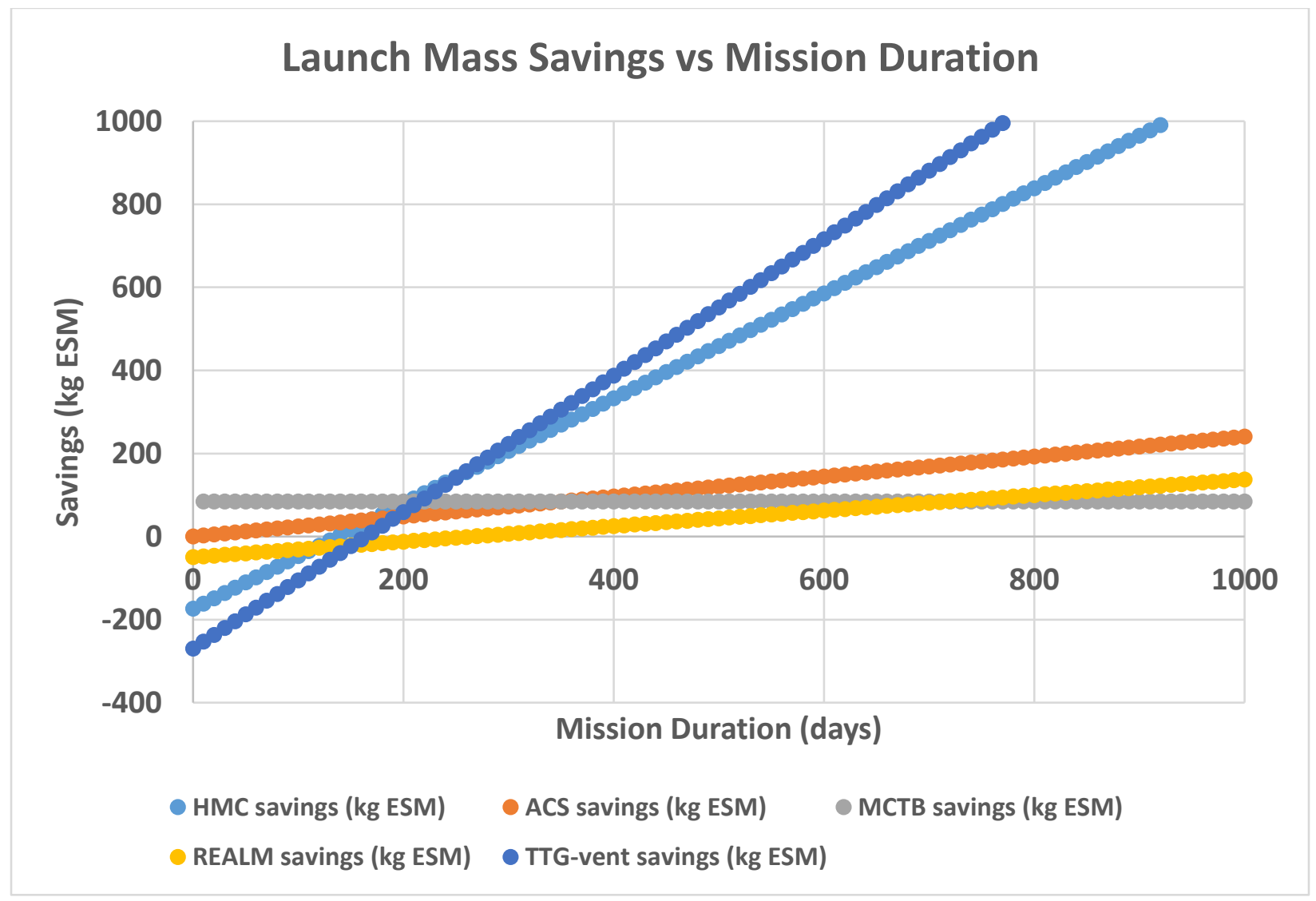

Fig. 9 Launch mass savings for various Logistics Reduction technologies using equivalent system mass.

\section{Acknowledgments}

The authors are extremely grateful for the spares analysis provided by Felipe Escobar of Binera at NASA Langley Research Center. The authors would also like to thank Pat Fink at NASA Johnson Space Center for his help in estimating the REALM benefits.

\section{References}

[1] Owens, A., et al., "Comparison of Spares Logistics Analysis Techniques for Long Duration Human Spaceflight," 45th International Conference on Environmental Systems, 12-16 July 2015, Bellevue, Washington, ICES-2015-288.

[2] Owens, A., et al.. "Supportability Challenges, Metrics, and Key Decisions for Future Human Spaceflight," AIAA SPACE and Astronautics Forum and Exposition, AIAA 2017-5124.

[3] Owens, A.C. and de Weck, O.L., "International Space Station Operational Experience and its Impacts on Future Mission Supportability," 48th International Conference on Environmental Systems, 8-12 July 2018, Albuquerque, New Mexico, ICES2018-198.

[4] Broyan, J.L., Schlesinger, T. and Ewert, M.K., "Exploration Mission Benefits From Logistics Reduction Technologies," 46th International Conference on Environmental Systems, 10-14 July 2016, Vienna, Austria, ICES-2016-140.

[5] Global Exploration Roadmap, Jan 2018, https://www.nasa.gov/sites/default/files/atoms/files/ger_2018_small_mobile.pdf

[6] Sargusingh, M. J., Anderson, M. S., Broyan, J. L., Macatangay, A. V., Shaw, L. A., Perry, J. L., Schneider, W. F., Gates, R. L., "NASA Environmental Control and Life Support Technology Development and Maturation for Exploration: 2017 to 2018 Overview," 48th International Conference on Environmental Systems, 8-12 July 2018, Albuquerque, New Mexico, ICES-2018182.

[7] Serio, M. A., Wojtowicz, M. A., Cosgrove, J. E., Stapleton, T. J., Torres, M. A., Ewert, M. K., Lee, J., “A Prototype Torrefaction Processing Unit (TPU) for Solid Human Waste," $48^{\text {th }}$ International Conference on Environmental Systems, 8-12 July 2018 , Albuquerque, New Mexico, ICES-2018-250. 
[8] Lee, J. M., Fisher, J. W., Pace, G., "Heat Melt Compactor Development," $48^{\text {th }}$ International Conference on Environmental Systems, 16-20 July 2017, Charleston, South Carolina, ICES-2017-267.

[9] https://www.fbo.gov/index?s=opportunity\&mode=form\&tab=core\&id=02c782d135f3d5414905697e89ef4473

[10]Anthony, S.M. and Hintze, P.E., "Trash-to-Gas: Determining the Ideal Technology for Converting Space Trash into Useful Products," 44th International Conference on Environmental Systems, ICES-2014-016.

[11]Ewert, M.K., et al., "Comparing Trash Disposal and Reuse Options for Deep Space Gateway and Mars Missions," AIAA Space 2017, AIAA 2017-5126.

[12]Byrne, V., et al., “Advanced Exploration Systems (AES) Logistics Reduction and Repurposing Project: Advanced Clothing Ground Study Final Report”, NASA JSC report \#CTSD-ADV-1088, Nov. 5, 2013.

[13]Poritz, et al., "Advanced Exploration Systems (AES) Logistics Reduction Project: Intravehicular (IVA) Clothing Study", NASA JSC report \#CTSD-ADV-1257, Nov. 20, 2015.

[14]Fink, P.W., et al., “Autonomous Logistics Management Systems for Exploration Missions,” AIAA Space 2017, AIAA 20175256.

[15]Levri, J.A., et al., “Advanced Life Support Equivalent System Mass Guidelines Document,” NASA/TM-2003-212278.

[16]Simon, M., Latorella, K., Martin, J., Cerro, J., Lepsch, R., Jefferies, S., and Goodliff, K., "NASA's Advanced Exploration Systems Mars Transit Habitat Refinement Point of Departure Design,” 2017 IEEE Aerospace Conference, Big Sky, MT, 2017.

[17]Ewert, et al., "Comparing Trash Disposal to Use as Radiation Shielding for a Mars Transit Vehicle," 47 ${ }^{\text {th }}$ International Conference on Environmental Systems, ICES-2017-178. 